\title{
Ataque de Aegorhinus nodipennis (Curculionidae) en forestaciones y huertas de estancias de Tierra del Fuego: ¿una invasión incipiente?
}

\author{
Aegorhinus nodipennis (Curculionidae) attack in forest plantations and \\ orchards of Tierra del Fuego ranches: an incipient invasion?
}

María Vanessa Lencinas ${ }^{1}$, Francisco José Zamora² \& Guillermo José Martínez Pastur ${ }^{3}$

Las invasiones biológicas se caracterizan por la capacidad de crecimiento poblacional, gran dispersión en el corto plazo, y amenaza sobre el ecosistema original y las especies nativas por parte de la especie invasora (Alpert et al. 2000). Son reconocidas mundialmente como causantes de buena parte de las extinciones modernas (54\%), siendo las islas particularmente vulnerables (Sodhi et al. 2009). En Tierra del Fuego, se han detectado aproximadamente unas 30 especies exóticas con comportamiento invasor, entre las cuales sólo unas pocas son insectos. En la mayoría de estos casos, tanto la presencia como la dispersión suelen estar asociadas a las ciudades y a las poblaciones humanas, como ocurre con las plagas urbanas de la chinche de la cama (Cymex lectularius Linnaeus 1758) y la cucaracha rubia (Blattella germanica Linnaeus 1767) en las ciudades de Ushuaia y Río Grande (obs. personal). Otras, han logrado establecerse con éxito en ambientes naturales y dispersarse hasta lugares recónditos, como ha ocurrido con la chaqueta amarilla (Vespula Thomson 1869 spp.) (Sola et al. 2015; Rendoll Cárcamo et al. 2016) y el abejorro (Bombus Latreille 1802 spp.) (Morales et al. 2016; Rendoll Cárcamo et al. 2017). En el caso de las plagas (frutihortícolas, forestales), suelen asociarse específicamente a un rango limitado de plantas hospederas, siendo relativamente bajo el riesgo de dispersión sobre los ecosistemas naturales donde esas especies naturalmente no ocurren. Por ejemplo, el pulgón gigante del sauce (Tuberolachnus salignus Gmelin 1790) ha sido detectado sobre 50 especies de sauces en Nueva Zelanda, y sólo en una especie nativa (Sopow et al. 2017). La capacidad de una plaga o patógeno no autóctono de afectar los ecosistemas forestales nativos depende fundamentalmente de su modo de acción (sobre qué parte de la planta y cómo actúa), de la especificidad sobre el hospedero (cuán amplia es su tolerancia a utilizar distintos huéspedes, edades o tamaños) y de su virulencia (con cuánta velocidad produce la muerte de las plantas) (Lovett et al. 2006).

Sin embargo, es posible que las especies nativas de una región, o incluso endémicas, también actúen como invasoras (Alpert et al. 2000). Esto suele ocurrir cuando ingresan a regiones fuera de su área de distribución natural, o ambientes donde no suelen estar presentes, pero donde determinadas condiciones les son propicias, incluso más que en su hábitat natural. Por ejemplo, por ausencia de depredadores o por mayor oferta de alimento. Respecto de los artrópodos, existen especies nativas que pueden actuar como

1 Centro Austral de Investigaciones Científicas (CADIC-CONICET). Houssay 200. (9410) Ushuaia,

Tierra del Fuego, Argentina.

$\square$ mulencinas@conicet.gov.ar

2 Agrónomo General Titular de Servicios Agropecuarios Fueguinos. Reverendo Padre Forgacs 2236. (9420) Río Grande, Tierra del Fuego, Argentina. zamorataruca@yahoo.com.ar

3 Centro Austral de Investigaciones Científicas (CADIC-CONICET). Houssay 200. (9410) Ushuaia, Tierra del Fuego, Argentina. gpastur@conicet.gov.ar 
invasoras o plagas sobre plantas hospederas que no son nativas, sino introducidas y cultivadas por el hombre dentro del área de distribución natural del artrópodo, sobre las que se comportan como plagas por ser más fácilmente utilizables o colonizables, y ocupar grandes superficies con muchos individuos, conformando cultivos monoespecíficos. Algunos de estos ejemplos son varias especies de Aegorhinus Erichson 1834 sobre cultivos frutihortícolas, como el cabrito de los frambuesos (A. superciliosus Guérin-Méneville 1830) (Parra et al. 2009) y el cabrito del duraznero (A. phaleratus Erichson 1834) (Caballero, 1972).

Sobre la base de estas consideraciones, los objetivos de esta nota son: (i) presentar los primeros registros en Tierra del Fuego del curculiónido Aegorhinus nodipennis Hope 1834 ("cabrito del ciruelo" o "cabrito de los arándanos"); (ii) describir los daños y las especies hospederas sobre las que fue observado; y (iii) discutir y alertar sobre su potencial invasor sobre la vegetación nativa de Fuegia-Magallanes, en relación al conocimiento previo disponible sobre esta especie.

La presencia del curculiónido A. nodipennis fue detectada por primera vez en Tierra del Fuego en diciembre de 2017 (Tabla 1), por uno de los autores de esta nota (F. J. Zamora), quien aportó fotos y más de 20 ejemplares, entre adultos $e$ inmaduros en estado larvario de la especie mencionada, habiendo sido identificada por M. V.
Lencinas. Su ataque se observó específicamente en el casco de Ea. Sara $(-53,446279$ LS; $-68,170188$ LW) (Fig. 1), tanto en los alrededores de la casa principal como en la huerta y en el camino de acceso, a lo largo de una cortina forestal.

Posteriormente, en febrero de 2021, se detectó nuevamente a esta especie en árboles exóticos implantados en el casco de la Ea. Viamonte (-53,994871 LS; -67,413598 LW) (Fig. 1), corroborándose su identidad sobre la base de fotografías (Tabla 1, Fig. 2). Durante el mismo mes, en Ea. Sara, otros ejemplares fueron observados por uno de los autores (F. J. Zamora), en cortinas forestales y en la huerta. Posteriormente, en el mes de marzo, se verificó la presencia en Ea. Viamonte por observación directa y colecta manual de ejemplares adultos (dos individuos). Luego, mediante monitoreos con trampas tipo pitfall que se revisaron cada 15 días, se registró la emergencia de adultos hasta mediados de abril de 2021. En total, fueron colectados seis individuos adultos mediante pitfall y tres manualmente. Cabe mencionar que el bajo número de capturas probablemente estuvo vinculado a que sólo se instalaron dos trampas cercanas a los árboles afectados, priorizándose el monitoreo sobre el bosque nativo en los alrededores del casco (con 10 trampas). Asimismo, se realizó una nueva visita a Ea. Sara a fines de marzo de 2021, donde

Tabla 1. Registros de Aegorhinus nodipennis (Curculionidae) en Tierra del Fuego.

\begin{tabular}{|c|c|c|c|c|}
\hline Sitio & Fecha & Registro & $\begin{array}{l}\text { Ambiente/ } \\
\text { Hospedero }\end{array}$ & $\begin{array}{l}\text { Observador/ } \\
\text { Colector }\end{array}$ \\
\hline Ea. Sara & Dic 2017 & $\begin{array}{l}\text { Registro fotográfico, observa- } \\
\text { ción y colecta }\end{array}$ & $\begin{array}{l}\text { Casco de estancia. Abedul, } \\
\text { sauce, serbal }\end{array}$ & F. J. Zamora \\
\hline Ea. Viamonte & Feb 2021 & Registro fotográfico & Casco de estancia. Manzano & M. C. Goodall \\
\hline Ea. Sara & Feb 2021 & Observación & $\begin{array}{l}\text { Casco de estancia. } \\
\text { Sauce, frutilla }\end{array}$ & F. J. Zamora \\
\hline Ea. Viamonte & Mar 2021 & Observación y colecta & $\begin{array}{l}\text { Casco de estancia. } \\
\text { Sauce, serbal, abedul }\end{array}$ & $\begin{array}{l}\text { M. V. Lencinas, } \\
\text { R. Soler }\end{array}$ \\
\hline Ea. Sara & Mar 2021 & $\begin{array}{l}\text { Observación, colecta y regis- } \\
\text { tro fotográfico }\end{array}$ & $\begin{array}{l}\text { Casco de estancia. } \\
\text { Sauce }\end{array}$ & $\begin{array}{l}\text { M. V. Lencinas, } \\
\text { G. J. Martínez Pastur }\end{array}$ \\
\hline Ea. Viamonte & Abr 2021 & Observación y colecta & $\begin{array}{l}\text { Casco de estancia. } \\
\text { Sauce, serbal }\end{array}$ & M. V. Lencinas \\
\hline
\end{tabular}




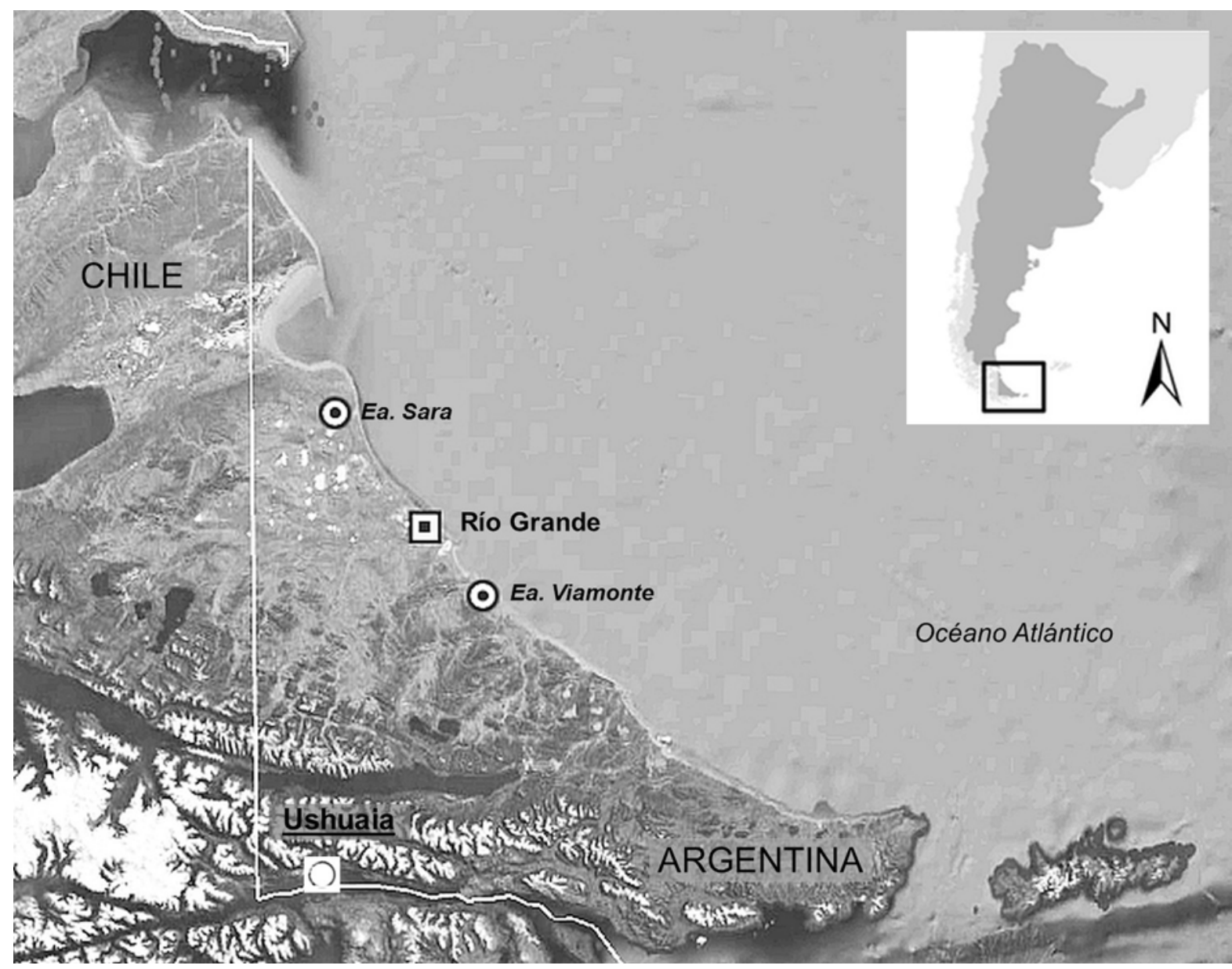

Fig. 1. Mapa de Tierra del Fuego (Argentina), con la ubicación de las estancias (Sara y Viamonte) y la ciudad de Río Grande, donde fue observado Aegorhinus nodipennis (Curculionidae). Se indica también la ubicación de la capital de la provincia, Ushuaia.

también se colectaron manualmente numerosos individuos (29 adultos y 2 larvas) (Tabla 1). En la misma recorrida, se visitaron los cascos de otras estancias cercanas a Ea. Sara (Ea. María Behety y Ea. José Menéndez), donde no se pudo constatar la presencia de A. nodipennis. Todos los individuos colectados por los autores de esta comunicación, así como los registros fotográficos, están depositados en la Colección Entomológica del CADIC.

Por otra parte, vale aclarar que, en abril de este año, personal de SENASA (Servicio Nacional de Sanidad y Calidad Agroalimentaria) pudo constatar la presencia de A. nodipennis en el vivero municipal de la ciudad de Río Grande (Fig. 1). Se colectó un adulto que fue identificado por $\mathrm{M}$. V. Lencinas y fue devuelto a SENASA.
Los ataques registrados en Ea. Sara provocaron la mortalidad de gran cantidad de árboles adultos de varias especies exóticas implantadas con valor paisajístico y ornamental, algunos muy añosos y de gran importancia para los dueños de las estancias, ya que habían sido traídos desde Europa por los fundadores, en los orígenes de los establecimientos (Fig. 3). Estos fueron principalmente árboles de madera blanda, como abedules (Betula pendula Roth 1788) y olmos (Ulmus minor Mill. 1768), habiendo sido también atacadas y diezmadas las cortinas forestales de sauces (Salix L. 1764 spp.). Se encontraron ejemplares (adultos y larvas) en la madera del cuello y de la base de las plantas atacadas, con gran profusión de galerías superficiales, madera en descomposición y orificios de salida de adultos 

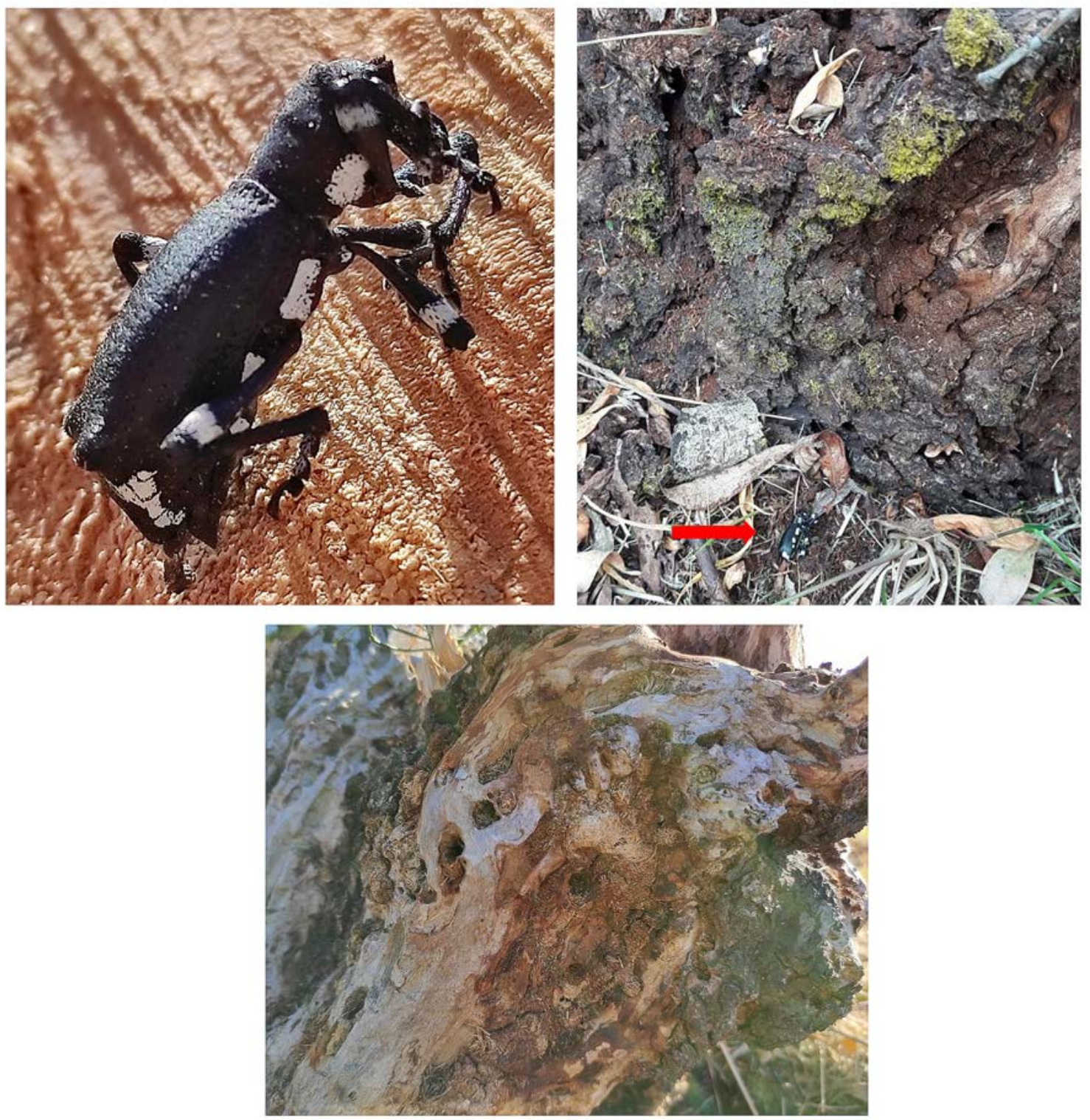

Fig. 2. Ejemplar de Aegorhinus nodipennis (Curculionidae) y daños en sauce (orificios de salida, galerías, descortezado). La flecha roja señala un individuo en la base del árbol (fotos M. V. Lencinas y G. J. Martínez Pastur).

(Fig. 2). En diálogo con los encargados del parque y la huerta, también fue confirmada la observación de adultos en grandes cantidades sobre frutillas (Fragaria L. 1753 sp.), donde realizaron cortes en las hojas cercanos a su punto de inserción en el tallo, generando la mortalidad de las plantas en un amplio sector del cultivo.

En la Ea. Viamonte, también se observaron ataques en ejemplares de especies exóticas ornamentales de madera blanda, en el parque que rodea el casco de la estancia, tanto sauces y abedules, como serbales (Sorbus aucuparia L. 1753), con abundantes perforaciones y signos evidentes de decaimiento, deterioro avanzado y/o muerte de ramas o fustes completos. Y aunque no se observaron daños en los árboles, también se encontraron adultos en manzanos (Malus Mill. 1764 sp.) y cerezos (Prunus cerasus L. 1753). El análisis detallado de sauces afectados permitió observar una gran afectación a la altura 
del cuello de la planta, con numerosos orificios (mayormente por debajo de los $50 \mathrm{~cm}$ de altura), residuos finos tipo aserrín y otro material leñoso en descomposición de mayor tamaño, y rastros de galerías superficiales. En algunos troncos, pudieron observarse individuos adultos recientemente emergidos y emergiendo a través de los orificios de salida de las cámaras de pupación (Fig. 2).

Aegorhinus nodipennis es una especie nativa de Argentina y Chile, específicamente de la Patagonia continental, siendo comúnmente observada en la zona del Maule y de los Bosques Valdivianos en este último país (Morrone \& Roig Juñent, 1995), y en el Norte cordillerano de la provincia de Santa Cruz en Argentina (obs. personal). En Chile, esta especie está considerada una de las mayores pestes para la fruticultura, sobre todo por su impacto negativo sobre la producción de avellanas (Corylus avellana $\mathrm{L}$. 1753) en la región de la Araucanía (Zavala et al. 2011), ya que mata los árboles que ataca. En esta zona de distribución, ha sido observada utilizando como hospederos al maitén (Maytenus boaria Molina 1782), al roble pellín (Nothofagus obliqua
(Mirb.) Oerst. 1872), al sauce o mimbrera blanca (Salix vimminalis L. 1753), al maqui (Aristotelia chilensis (Molina) Stuntz 1914), al arándano azul (Vaccinium corymbosum L. 1753), al abedul, al avellano chileno (Gevuina avellana Molina 1782), al boldo (Peumus boldus Molina 1782), al radal (Lomatia hirsuta Diels ex J. F. Macbr. 1937), al canelo (Drimys winteri J. R. Forst. \& G. Forst. 1776) y a la lenga (Nothofagus pumilio (Poepp. \& Endl.) Krasser 1896). Asimismo, cabe mencionar que estos autores también señalan a $A$. nodipennis como distribuido en áreas fuertemente dominadas por matorrales arborescentes, por lo que no se puede descartar que utilice otros hospederos no arbóreos para su desarrollo.

El conocimiento sobre el ciclo biológico y su actividad estacional se basa en información producida en Chile en la zona de donde es originario (Cisternas et al. 2016), pero la observación de ejemplares adultos y en distinto estado larvario de desarrollo en Tierra del Fuego indica la capacidad de la especie para completar su ciclo y reproducirse, al menos en las condiciones microclimáticas de los últimos cinco años, y en los hospederos en

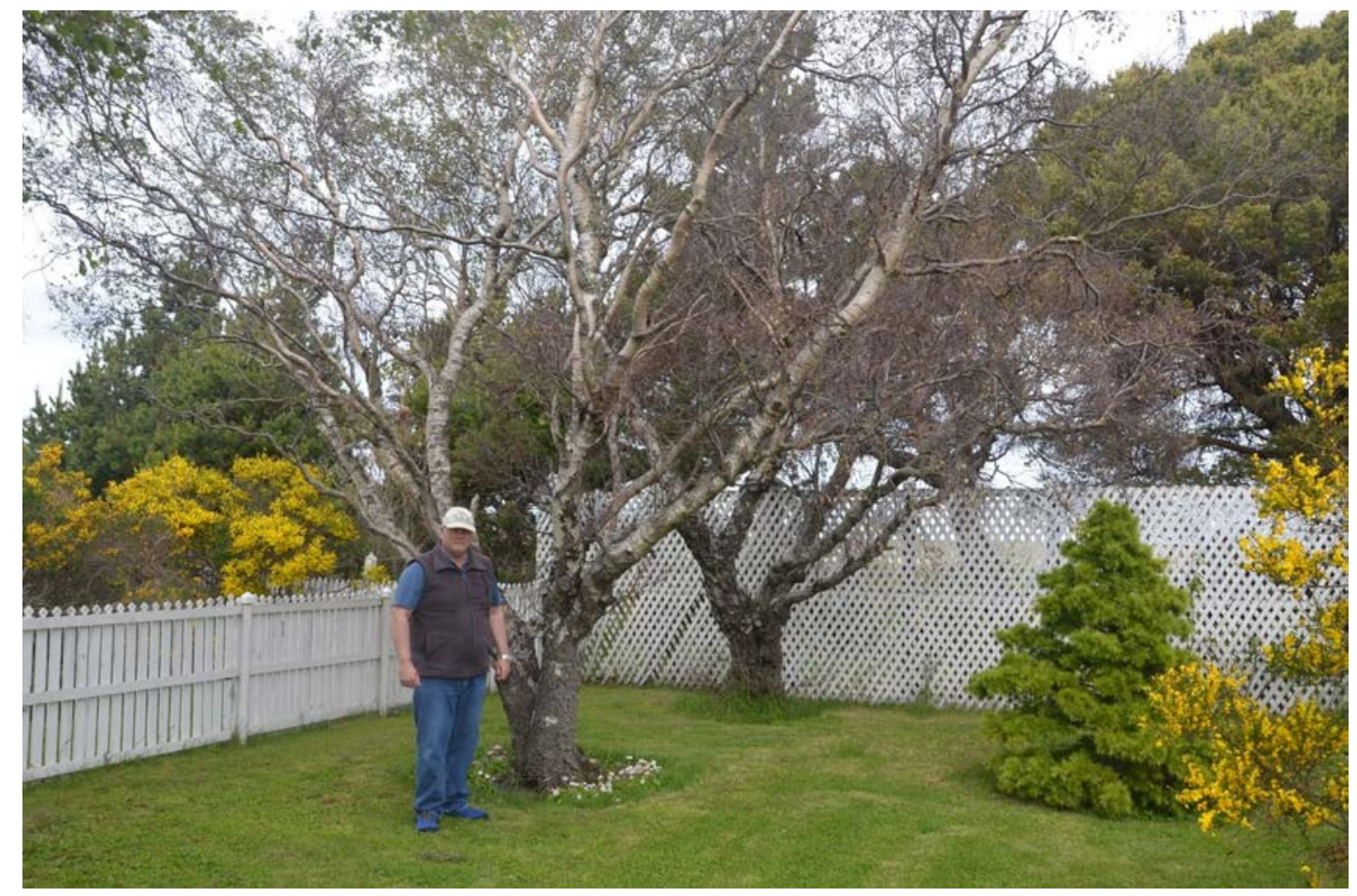

Fig. 3. Abedul afectado por Aegorhinus nodipennis (Curculionidae) en el parque de Ea. Sara, con ramas muertas y gran parte de la copa senescente (foto F. J. Zamora). 
los que fue directamente observada atacando el cuello de la planta y sus raíces (e.g., sauces y abedules). Es decir, que ya ha superado las etapas de arribo y establecimiento, pudiendo también haber comenzado con la dispersión (Liebhold et al. 1995). Por otra parte, cabe mencionar que en 2017 y en 2021, se realizaron consultas a SENASA respecto de si esta especie era considerada plaga en Argentina, y el SINAVIMO (Sistema Nacional Argentino de Vigilancia y Monitoreo de plagas, https://www.sinavimo.gob.ar// respondió que no ha sido aún catalogada como tal. Esto difiere de la catalogación que recibe en Chile, donde la especie es considerada una plaga de importancia para cultivos frutihortícolas (Zavala et al. 2011), aunque no para forestaciones. Sin embargo, su presencia y daños en especies ornamentales, forestales y frutihortícolas de Tierra del Fuego han sido recientemente declarados ante SENASA.

$\mathrm{Si}$ bien $A$. nodipennis parecería, de momento, estar únicamente asociada a ambientes con presencia humana (porque solamente se ha registrado en asociación a árboles exóticos en cascos de estancias o en viveros), existe la posibilidad de que pueda sobrevivir en áreas naturales de todo el archipiélago fueguino. Siendo una especie que puede desarrollarse en una gran variedad de hospederos, entre los cuales se incluyen según observaciones en su lugar de origen, algunas de las especies nativas de los bosques magallánicos (e.g. lenga y canelo, según Zavala et al. 2011), el hallazgo de poblaciones relativamente estables de A. nodipennis en forestaciones de cascos de estancias de Tierra del Fuego argentina, con cierta capacidad reproductiva y de dispersión, genera un gran riesgo potencial para el bosque nativo.

Aunque se desconoce cómo arribó esta plaga, una de las posibles vías de introducción de la especie sería aquella asociada a la producción de animales y plantas vivos (NISC, 2006), tal como plantas de vivero procedentes de la zona de distribución natural de A. nodipennis (e.g., El Bolsón, en la provincia de Río Negro), o tierra de esa zona, que pudieran haber sido adquiridas por particulares o viveristas, y trasladadas hasta Tierra del Fuego. Aunque no existen evidencias ni registros que permitan constatar específicamente cómo llegó a ambos cascos de estancias (distanciados alrededor de $80 \mathrm{~km}$ en línea recta entre sî), su presencia corroborada en ambos sitios, $y$ en uno de ellos su permanencia por al menos los últimos 4 años (desde 2017 a 2021), genera una gran incertidumbre respecto del potencial de invasión y de afectación de nuestros ecosistemas naturales; esto, principalmente, debido a que se desconoce la existencia en Tierra del Fuego de depredadores naturales que pudieran controlarlo, la velocidad para cumplimentar su ciclo biológico a estas latitudes (e.g. duración total del ciclo, cantidad de generaciones anuales, estacionalidad), la capacidad de utilización de vegetación no arbórea (la cual es mencionada como posibilidad por otros investigadores en su área de distribución natural), su habilidad de dispersión (en el casco de Ea. Sara se registró en sectores distantes $150 \mathrm{~m}$ entre sí) y el nivel de daño que podría generar sobre las especies arbóreas nativas si logra arribar al bosque, por sí misma o a través de sinergias negativas con otros insectos que las afectan usualmente (e.g. escolítidos, cerambícidos).

Cabe mencionar que el casco de Ea. Viamonte está instalado junto a un antiguo parche de ñire ( $N$. antarctica (G.Forst.) Oerst. 1871) que actualmente rodea la casa, la huerta y la parquización donde $A$. nodipennis fue observado, y que durante este verano (marzo a abril de 2021), se realizó un monitoreo periódico cada 15 días con trampas pitfall para evaluar la presencia de A. nodipennis, sin que se haya podido colectar ningún ejemplar en el bosque nativo. Esto podría indicar que el ñire no es un buen hospedero para A. nodipennis (tampoco ha sido identificado como hospedero en el área de distribución original de la especie), probablemente por la dureza de la madera y la corteza de gran grosor que caracteriza a estas plantas en estado de madurez avanzada. Sin embargo, dicha situación podría cambiar frente a la falta de otros recursos, o en ejemplares jóvenes con corteza de menor grosor, lo cual debería ser más profundamente estudiado. En el caso de la lenga, que sí ha sido descrita como hospedero en el área de origen, el bosque más cercano de esta especie arbórea se encuentra aproximadamente a 10 $\mathrm{km}$ del casco de Ea. Viamonte, desconociéndose si es posible que la especie se disperse por sus propios medios a esas distancias. Asimismo, se observa que en la región donde $A$. nodipennis es originaria, los bosques son multiespecíficos, 
mientras que en Tierra del Fuego son mayormente monoespecíficos, lo cual podría significar un mucho mayor nivel de afectación y riesgo por la cercanía y mayor disponibilidad de plantas hospederas, si este curculiónido arribara hasta ellos.

Por otra parte, si bien en ambas estancias se realizaron controles desde los primeros momentos en que se detectó el daño sobre los árboles, incluyendo la fumigación con productos químicos (sistémicos y de contacto), y la remoción y quema de los individuos arbóreos afectados, es evidente la dificultad para controlarlo, ya que los huevos se depositan en el suelo y las larvas rápidamente penetran dentro de las plantas una vez que eclosionan, siendo muy difícil que entren en contacto con los plaguicidas utilizados. Por otro lado, la emergencia de adultos parecería ocurrir durante un largo período de tiempo durante la estación de crecimiento. Esta falta de concentración en el momento de aparición de los adultos, así como las difíciles y muchas veces extremas condiciones climáticas que dificultan la aplicación de fumigaciones (por excesivo viento o lluvias), contribuyen a que el control sobre esta especie, de momento, haya sido poco eficiente.

Dada la información recolectada hasta ahora, resulta de gran importancia continuar el monitoreo y reforzar el control para minimizar la dispersión de esta especie, tanto por investigadores y técnicos, como por autoridades de aplicación (e.g. SENASA, autoridades provinciales y municipales), gestores ambientales, organizaciones de la sociedad civil (e.g. ONGs, Asociación Rural de Tierra del Fuego) y la comunidad en general. No sólo para que no se disperse por ecosistemas nativos, sino también para reducir su impacto sobre las parquizaciones en los cascos de estancias, principalmente aquellos que constituyen una herencia cultural valiosa, de gran importancia para la historia de la población fueguina.

\section{AGRADECIMIENTOS}

A los dueños de Ea. Sara, Viamonte, María Behety y José Menéndez, que nos permitieron explorar sus establecimientos e instalar sistemas de monitoreo para evaluar la evolución de la presencia y la abundancia de Aegorhinus nodipennis.

\section{LITERATURA CITADA}

Alpert, P., Bone, E., y Holzapfel, C. (2000). Invasiveness, invasibility and the role of environmental stress in the spread of non-native plants. Perspectives in Plant Ecology, Evolution and Systematics, 3/1, 52-66.

Caballero, V. C. (1972). Some aspects of the bionomics of Aegorhinus phaleratus Erichson (Coleoptera, Curculionidae), on peach trees in Chile. Revista Peruana de Entomología, 15, 186-189.

Cisternas, E., France I., A., y Morán, V. A. (2016). Ciclo y actividad estacional de Aegorhinus nodipennis: en huertos de arándanos. INIA La Cruz, Informativo, 35.

Liebhold, A. M., Macdonald, W. L., Bergdahl, D., y Maestro, V. C. (1995). Invasion by exotic forest pests: a threat to forest ecosystems. Forest Science, 41, 1-49.

Lovett, G. M., Canham, C. D., Arthur, M. A., Weathers, K. C., y Fitzhugh, R. D. (2006). Forest ecosystem responses to exotic pests and pathogens in eastern North America. BioScience, 56, 395-405.

Morales, C. L., Montalva, J., Arbetman, M., Aizen, M. A., Smith-Ramírez, C., Vieli, L., y Hatfield, R. (2016). Bombus dahlbomii. The IUCN Red List of Threatened Species 2016: e.T21215142A100240441, Gland, Switzerland.

Morrone, J. J., y Roig Juñent, S. (1995). The diversity of Patagonian Weevils. An illustrated checklist of the Patagonian Curculionoidea (Insecta: Coleoptera). Buenos Aires: Ediciones L.O.L.A.

NISC (National Invasive Species Council) (2006). Focus Group Conference Report and Pathways Ranking Guide. June-August 2005. U.S. Department of Agriculture, Animal Plant Health Inspection Service, Plant Protection and Quarantine. Riverdale.

Parra B., L., Mutis T., A., Aguilera P., A., Rebolledo R., R., y Quiroz C., A. (2009). Estado del conocimiento sobre el cabrito del frambueso (CF), Aegorhinus superciliosus (Guérin) (Coleoptera: Curculionidae). IDESIA, 27(1), 57-65. 
Rendoll Cárcamo, J., Contador, T., Crego, R. D., Jordán, N. I., Schüttler, E., Gañán, M., Jiménez, J. E., Rozzi, R., Massardo, F., y Kennedy, J. H. (2016). Primer registro de Vespula vulgaris (Linnaeus 1758) (Hymenoptera: Vespidae) en la isla Navarino, Chile. Gayana, 80(1), 133-136.

Rendoll Cárcamo, J. A., Contador, T. A., Saavedra, L., y Montalva, J. (2017). First record of the invasive bumblebee Bombus terrestris (Hymenoptera: Apidae) on Navarino Island, southern Chile $\left(55^{\circ} \mathrm{S}\right)$. Journal of Melittology, 71, 1-5.

Sodhi, N. S., Brook, B. W., y Bradshaw, C. A. J. (2009). Causes and consequences of species extinctions. In S. A. Levin (Ed.), Princeton Guide to Ecology. Nueva Jersey: Princeton University Press.

Sola, F. J., Valenzuela, A. E. J., Anderson, C.
B., Martínez Pastur, G. J., Lencinas, M.V. (2015). Reciente invasión del Archipiélago de Tierra del Fuego por la avispa Vespula germanica (Hymenoptera: Vespidae), Revista de la Sociedad Entomológica Argentina, 74(3-4), 197-202.

Sopow, S. L., Jones, T., Mclvor, I., McLean, J. A., y Pawson, S. M. (2017). Potential impacts of Tuberolachnus salignus (giant willow aphid) in New Zealand and options for control. Agricultural and Forest Entomology, http://doi.org/10.1111/afe.12211

Zavala, A., Elgueta, M., Abarzúa, J., Aguilera, A., Quiroz, A., y Rebolledo, R. (2011). Diversity and distribution of the Aegorhinus genus in the La Araucanía Region of Chile, with special reference to $A$. superciliosus and A. nodipennis. Ciencia e Investigación Agraria, 38(3), 367-377. 